\title{
In Situ Preparation of Core Shell-Polypyrrole /Poly (Acrylonitrile-Co-Vinyl Acetate) Nanoparticles and Their Nanofibers
}

\author{
Burcu Arman, A. Sezai Sarac* \\ Department of Chemistry, Polymer Science and Technology, Nanoscience \& Nanoengineering, Istanbul \\ Technical University, Istanbul, Turkey \\ Email: $\underline{\text { sarac@itu.edu.tr }}$
}

Received 12 February 2014; revised 12 March 2014; accepted 20 March 2014

Copyright (C) 2014 by authors and Scientific Research Publishing Inc.

This work is licensed under the Creative Commons Attribution International License (CC BY). http://creativecommons.org/licenses/by/4.0/

(c) (i) Open Access

\section{Abstract}

Poly (acrylonitrile-co-vinyl acetate)/polypyrrole composite particles with uniform size and morphology have been synthesized using one-step polymerization that involves swelling and coating of polypyrrole (PPy) into P (AN-co-VAc) latex nanoparticles. As an initial stage, free radical copolymerization of acrylonitrile (AN) and vinyl acetate (VAc) was synthesized by emulsion polymerization using ammonium persulfate (APS) and dodecyl benzene sulfonic acid salt (DBSA) as a surfactant. P (AN-co-VAc)/PPy composites were obtained first time by in situ addition of the pyrrole into the reaction medium. The electrospun $\mathrm{P}$ (AN-co-VAc)/PPy nanofibers were obtained from the nanoparticles with better properties and the effect of PPy on the morphology of nanofibers was studied by scanning electron microscopy (SEM). High degree of homogeneity and molecular order induced by molecular dispersion of polypyrrole on copolymer matrix without phase separation improve the transport properties and stability of polypyrrole, which are critical for high-performance organic electronics.

\section{Keywords}

Poly (Acrylonitrile-Co-Vinyl Acetate), Polypyrrole, Emulsion Polymerization, Electrospinning, Nanofiber

\section{Introduction}

Conjugated polymers are often brittle in nature and hence very difficult to make a film from them. In order to

\footnotetext{
"Corresponding author.
}

How to cite this paper: Arman, B. and Sarac, A.S. (2014) In Situ Preparation of Core Shell-Polypyrrole/Poly (Acrylonitrile-Co-Vinyl Acetate) Nanoparticles and Their Nanofibers. Soft Nanoscience Letters, 4, 42-49. 
improve the processibility of conjugated polymers, they can be combined with mechanically stable insulating polymers in micro- or nano-scale through core-shell morphology. These composite particles may have manifold applications such as antistatic coating, dampers, electrodes, separation membranes, electrochromic devices, actuators, and the sensors.

These core-shell conducting composites have two advantages. Firstly, depending on the type of insulating polymer used as core, these conducting polymer-coated latex particles can exhibit very good mechanical stability and secondly the amount of conducting polymer used can be greatly reduced without much loss of conductivity [1] [2].

Related to its good intrinsic properties, polypyrrole has proven promising for several applications including batteries, super capacitors, electrochemical, biosensors, conductive textiles and fabrics, mechanical actuators, electromagnetic interference (EMI) shielding, antistatic coatings and drug delivery systems. The intrinsic properties of polypyrrole are highly dependent on polymerization conditions. Its conjugated structure-delocalized-electronic structure, limits the processability, flexibility, and strength. These limitations can be resolved by making composites [3] [4]. Polypyrrole-coated carbon nanotubes have exhibited enhanced electrical properties [5].

Incorporating a number of flexible comonomers weakens the dipolar interactions among $\mathrm{CN}$ and thereby decreases melting point or increases decomposition temperatures of the PAN-based copolymers. Hence, it is important to consider the effect of comonomer on the properties of PAN-based copolymers.

Conductive textiles exhibit a range of outstanding properties, from the electrical conductivity, to shielding of electromagnetic radiations, dissipation of microwave energy, etc. The advantages of conducting polymer composites as compared to metals include lighter weight, corrosion resistance, and flexibility and, they are good materials for eliminating electromagnetic interference shielding (EMI) with their relatively high dielectric permittivity and conductivity compared with other polymers

AN-VAc copolymers have the significant effect to their thermal behavior and are also of interest as precursors in the production of high strength carbon fibers. AN is copolymerized with one or two comonomers, particularly with vinyl acetate (VAc). The copolymer of AN and VAc can be used as either plastic (VAc $>15 \mathrm{wt} \%$ ) or microfibers (VAc < $15 \mathrm{wt} \%$ ). The presence of AN in the structure provides the copolymer with good processability, electrochemical and thermal stability; VAc provides the mechanical stability.

Efficient gel-state dye-sensitized solar cells using polymer gel electrolytes based on poly (acrylonitrile-covinyl acetate), in situ gelation of such electrolytes for gel-state Dye-Sensitized Solar Cells, and one-step fabrication, modification of poly (acrylonitrile-co-vinyl acetate) microsphere preparations were recently reported [6]-[8]. One-pot fabrication of Poly (acrylonitrile-co-vinyl acetate)/Ag composite microspheres and their possible applications as catalysts were also realized [9].

The electrospun P (AN-co-VAc)/PPy nanofibers were obtained from the nanoparticles and the effect of PPy on the morphology of nanofibers was studied by scanning electron microscopy (SEM) and molded films of $P$ (AN-co-VAc)/PPy were tested by Dynamic Mechanical Analysis (DMA). Electrochemical impedance measurements were realized from dissolved nanofibers in DMF.

Since homogeneity by molecular dispersion of polypyrrole on copolymer matrix without phase separation improve the transport properties and stability of polypyrrole, we have recently concentrated to study on the composites of polypyrrole in the matrix of copolymer of AN and VAc by polymerization of pyrrole in copolymer matrix without surfactant and mainly as solvent casted films [10] [11].

To obtain better homogeneity and controllable nanoscale synthesis of polypyrrole in the presence of freshly formed copolymer particles in emulsion, in this study, first free radical copolymerization of acrylonitrile (AN) and vinyl acetate (VAc) was realized by using ammonium persulfate (APS) in the presence of dodecyl benzene sulfonic acid salt (DBSA) as a surfactant and then polymerization of pyrrole under in situ conditions was performed in this reaction medium to obtain core-shell nanoparticles first time. P (AN-co-VAc)/PPy composite core shell nanoparticles were obtained at optimum VAc comonomer content of the matrix by one-step polymerization of swelling and coating of pyrrole (Py) onto P (AN-co-VAc) latex nanoparticles. Composites were characterized; later nanofibers of P (AN-co-VAc) copolymer and P (AN-co-VAc)/PPy composite nanoparticles were obtained by electrospinning.

The electrospun P (AN-co-VAc)/PPy nanofibers were obtained from the nanoparticles and the effect of PPy on the morphology of nanofibers was studied by scanning electron microscopy (SEM) and molded films of $P$ 
(AN-co-VAc)/PPy were tested by Dynamic Mechanical Analysis (DMA). Electrochemical impedance measurements were realized from dissolved nanofibers in DMF.

High degree of homogenity and molecular order induced by molecular dispersion of polypyrrole on copolymer matrix without phase separation improve the transport properties and stability of polypyrrole, which are critical for high-performance organic electronics. These fibers can be used as textile filtration nanofiber mats and for carbon nanofiber precursor in energy storage applications.

\section{Experimental}

\subsection{Materials}

Acrylonitrile (Dow, 99.5 > \%) vinyl acetate (Innovene, 99.5 > \%) were provided by the AKSA Acrylic Chemical Inc., Yalova, Turkey. Ammonium persulfate (APS) was Merck product. Sodium dodecyl benzene sulfonic acid salt was purchased from Sigma Aldrich. Dimethylformamide (DMF), methanol and ethanol were all Merck reagents. The Pyrrole (Py) was an Aldrich reagent. All these reagents were used as received.

\subsection{Synthesis of P (AN-Co-VAc) Copolymer}

The typical process for obtaining P (AN-co-VAc) latex is as follows: $10 \mathrm{~g}$ of a mixture of monomers of AN and VAc (70:30 wt AN:VAc) were dropped into the three-neck flask, the aqueous mixture was stirred for 20 minutes. Then, $0.5 \mathrm{~g}$ emulsifier sodium dodecyl benzene sulfonic acid (DBSA) was dissolved in $60 \mathrm{~mL}$ water. DBSA was added to a stirred aqueous solution. 20 minutes later, the dissolved $0.92 \mathrm{~g}$ APS in $20 \mathrm{~mL}$ distilled water was added to the flask. The copolymerization was carried out in a flask equipped with a stirrer (700 rpm) and a condenser, and the temperature was adjusted to $70 \mathrm{C}$ and continued for $3 \mathrm{~h}$ under stirring.

Coating of P (AN-co-VAc) Latex Particles with Polypyrrole (core shell structure) P (AN-co-VAc) latex mixture was cooled for $30 \mathrm{~min}$ at room temperature and then, was transferred to 5 round-bottom flasks at equal volume. Four flasks were separated for the coating processes. One of them is kept for comparison. Then, pyrrole monomer with different quantities was added dropwise to the each separated flasks at room temperature, and the reaction continued for 18 hours. Resulting composite nanoparticles in emulsion were precipitated by the addition of methanol. Each product was filtered using filter paper, for the separation of impurities and oligomers, the resultant products were washed with deionized water, and ethanol, dried at room temperature. Composite formation scheme and structure are shown in Figure 1.

\section{Preparation of Electrospinning Solutions}

Five series of P (AN-co-VAc)/PPy composite nanoparticles with different content of pyrrole dissolved in DMF at room temperature.

Each solution was stirred at room temperature with the speed of $300 \mathrm{rpm}$ at least 4 hour. The electrospinning apparatus consists of a syringe pump (NE-500 model, New Era Pump Systems, Inc., USA) with feeding rate from $5.5 \mu \mathrm{L} / \mathrm{h}$ to $400 \mathrm{~mL} / \mathrm{h}$, high voltage DC power supplier generating positive DC voltage up to $50 \mathrm{kV}$ (ES50 model, Gamma High Voltage Inc., USA) and a grounded collector covered with aluminum foil.

\section{Results and Discussions}

\subsection{Copolymer and Composite Characterization}

Spectroscopic analysis PAN shows its characteristic absorption peaks at $2243 \mathrm{~cm}^{-1}$ and $1451 \mathrm{~cm}^{-1}$, corresponding to $\mathrm{CN}$ stretching and $\mathrm{CH}$ bending, respectively. The $\mathrm{C}=\mathrm{O}$ stretching, $\mathrm{C}-\mathrm{O}-\mathrm{C}$ stretching and $\mathrm{C}-\mathrm{O}$ stretching vibration peaks can be observed at 1736, 1232, and $1022 \mathrm{~cm}^{-1}$, respectively for PVAc. The strong absorption bands for $\mathrm{P}$ (AN-co-VAc) are $\mathrm{C}=\mathrm{O}$ stretching $\left(1736 \mathrm{~cm}^{-1}\right)$ and $\mathrm{CN}$ stretching $\left(2243 \mathrm{~cm}^{-1}\right)$ (Figure 2). FTIR-ATR spectrums for both of nanofibers and granular form of composite nanoparticles are the same except the characteristic peak of DMF at $1666 \mathrm{~cm}^{-1}$ due to the carbonyl stretching mode which DMF was involved in the structure as a plasticizer.

The change of Py content in the composite was followed by the absorbance values of PPy characteristic C-N stretching peak at $1451 \mathrm{~cm}^{-1}$ (Figure 2). ${ }^{1} \mathrm{H}$ NMR spectrums of copolymer were obtained using a $250 \mathrm{MHz}$ Bruker AC Aspect 3000 NMR spectrometer. Figure 3 shows the ${ }^{1} \mathrm{H}-\mathrm{NMR}$ spectra of copolymer recorded in 


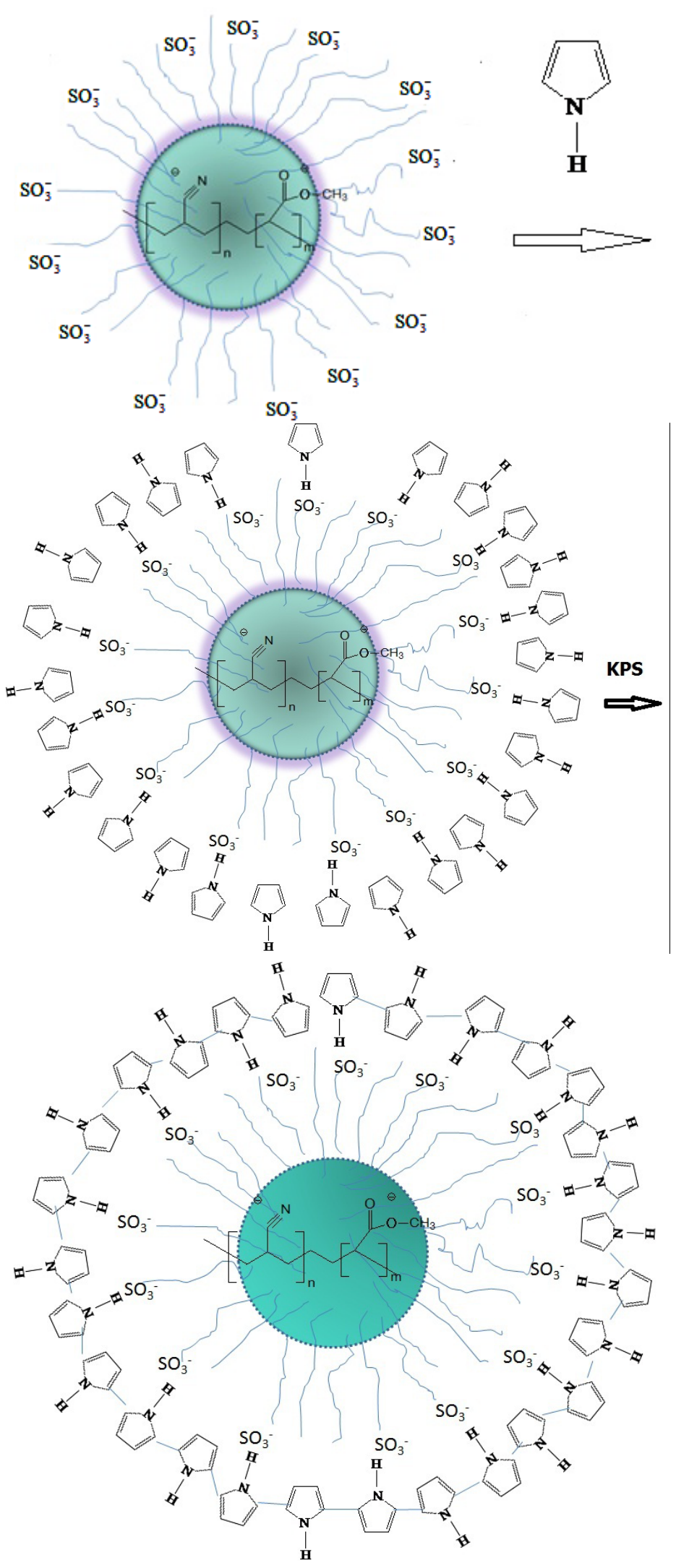

Figure 1. Scheme of core shell structure. 
Deuterated dimethyl sulphoxide (DMSO) using TMS as the internal standard. The peak at $2.07 \mathrm{ppm}$ is related to $-\mathrm{CH}_{2}$ protons of both AN and VAc and the peak at $3.12 \mathrm{ppm}$ is due to - $\mathrm{CH}$ proton of AN. The peak at $5.13 \mathrm{ppm}$ was assigned to - $\mathrm{CH}$ proton of VAc. These peaks are in agreement with literature [12]. The mole fraction of $\mathrm{P}$ (AN-co-VAc) was calculated from the ratio of the peak areas $5.13 \mathrm{ppm}$, corresponding to total peak area of 5.13 and 3. 12. Mol fraction of copolymer $[\mathrm{m} /(\mathrm{m}+\mathrm{n})]$ was calculated as 0.284 . Nanofibers dissolved in DMF were analyzed by UV-visible spectrophotometer. Nanofiber solution concentration was $0.11 \mathrm{wt} \%$ and kept constant for every measurement of composites. UV-Vis spectrum of polypyrrole shows an absorbance maxima at about $430 \mathrm{~nm}$, attributed to transitions of the valence to polaron state. A broad absorption peak was observed between $550 \mathrm{~nm}$ and $900 \mathrm{~nm}$, related to polaron to bipolaron transitions in PPy, in addition to the transition of pyrrole units with high conjugation.

Relationship between, FTIR-ATR and UV-Vis spectrophotometric results were presented in the Figure 2. C-N stretching peak of PPy shows its characteristic peak at $1451 \mathrm{~cm}^{-1}$. An increase in the FTIR absorbance values of PPy at $1451 \mathrm{~cm}^{-1}$ was observed corresponding to increase in PPy wt\% in feed ratio and the same trend was also observed in the UV-Vis spectrophotometric measurements by the increase in the absorbance of the peak of $430 \mathrm{~nm}$.

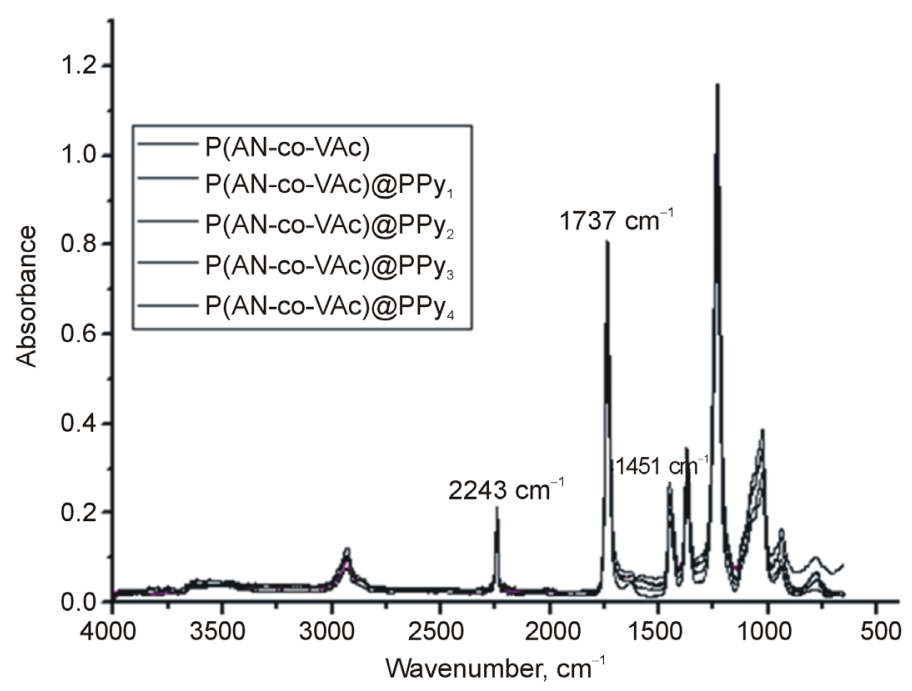

(a)

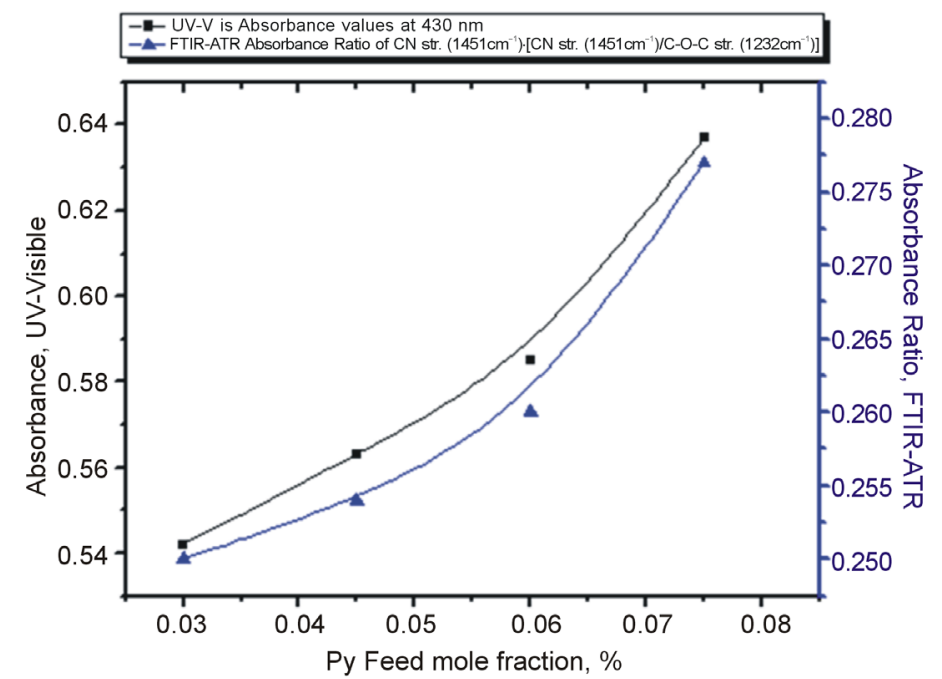

(b)

Figure 2. Absorbance of FTIR ATR and UV V is Spectrums vs initial pyrrole feed ratio for PPy/P (AN-co-VAc). 


\subsection{Viscosity and Particle Size Measurements}

The intrinsic viscosity of the polymer solutions in DMF was determined by Ubbelohde viscometer at $28 \mathrm{C}$ for $\mathrm{P}$ (AN-co-VAc) by DMF as a solvent. The molecular weight was calculated for P (AN-co-VAc) copolymer of 30 wt\% VAc feed ratio as $380 \mathrm{~kg} / \mathrm{mole}$ from intrinsic viscosity by using reported $\mathrm{K}$ and alpha values. A reduction in specific viscosities by the increase in pyrrole feed ratio was resulted. Lower viscosities are obtained for the composite solutions due to the presence of low molecular weight of conductive polymer. Latex form of $\mathrm{P}$ (AN-co-VAc) and P (AN-co-VAc)/PPy composites nanoparticle's morphology was investigated by SEM (Figure 3).
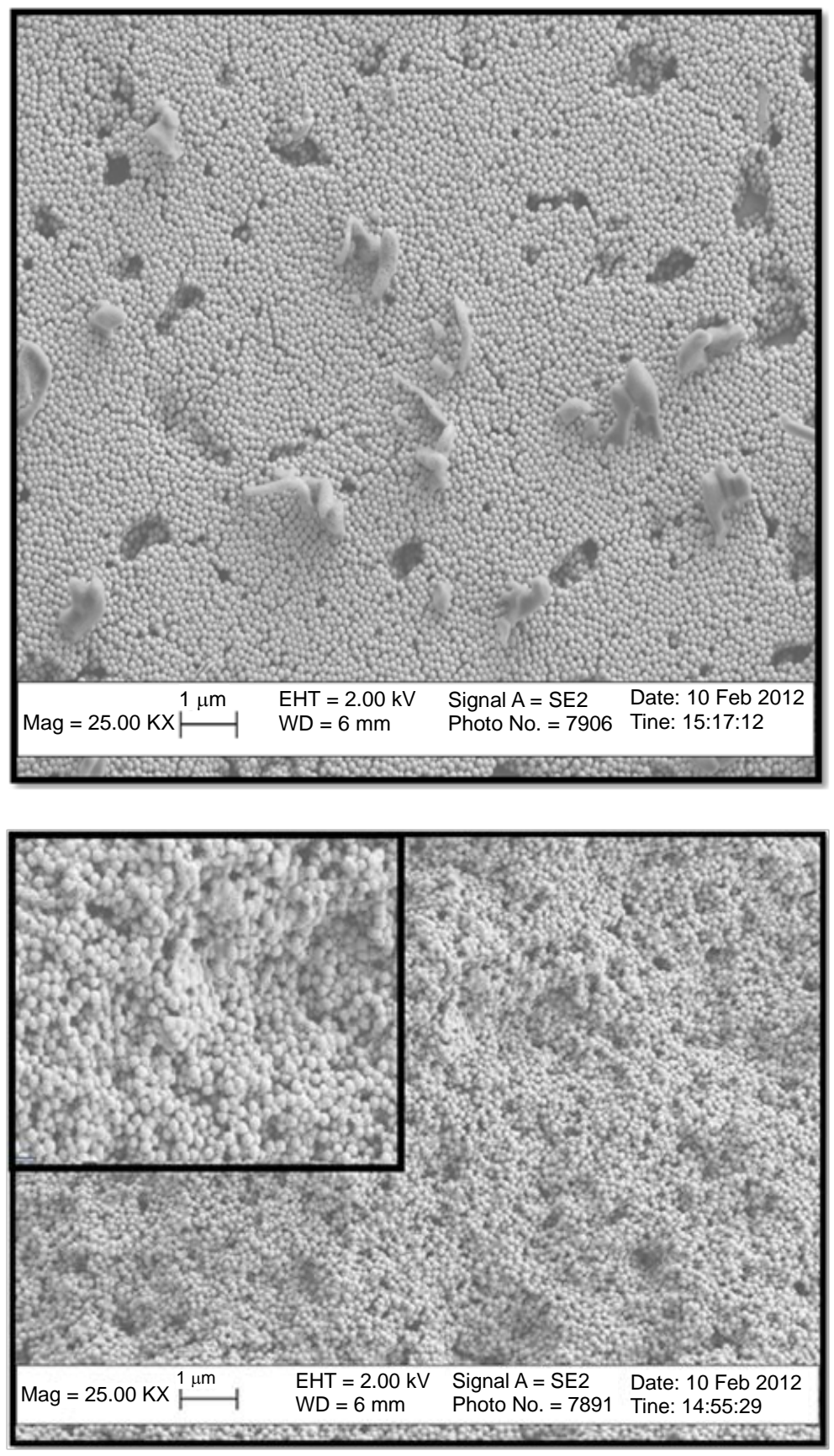

Figure 3. Latex form of P (AN-co-VAc) (upper) and P (AN-co-VAc)/PPy (lower-inset magnified) composites nanoparticle’s morphology investigated by SEM. 


\subsection{Morphology of Fibers}

The average diameter of electrospun fibers of different concentrations is determined by using Image $\mathrm{j}$ program to randomly measure the diameters of 40 individual fibers shown in SEM images with $\times 20,000$ magnitude (Figure 4). Mole percent of the initially added Py concentration varies from $\% 0.035-0.070$. The average diameters of the nanofibers are reduced from 200 to $120 \mathrm{~nm}$. In this study, elimination of surface roughness on nanofibers structure was due to the well interaction of PPy with matrix and well dispersion of latex particles in the DBSA medium resulting an improve in the solubility of PPy [10].

The relatively low molecular weight of conductive polymers, decrease in specific viscosity of composites resulted a small nanofiber diameters. Interaction of PPy with matrix creates a decrease in viscosity, and that causes the smaller diameter of nanofibers. Moreover, electrospinning solutions of nanofibers with small average diameters have exhibited higher conductivity.
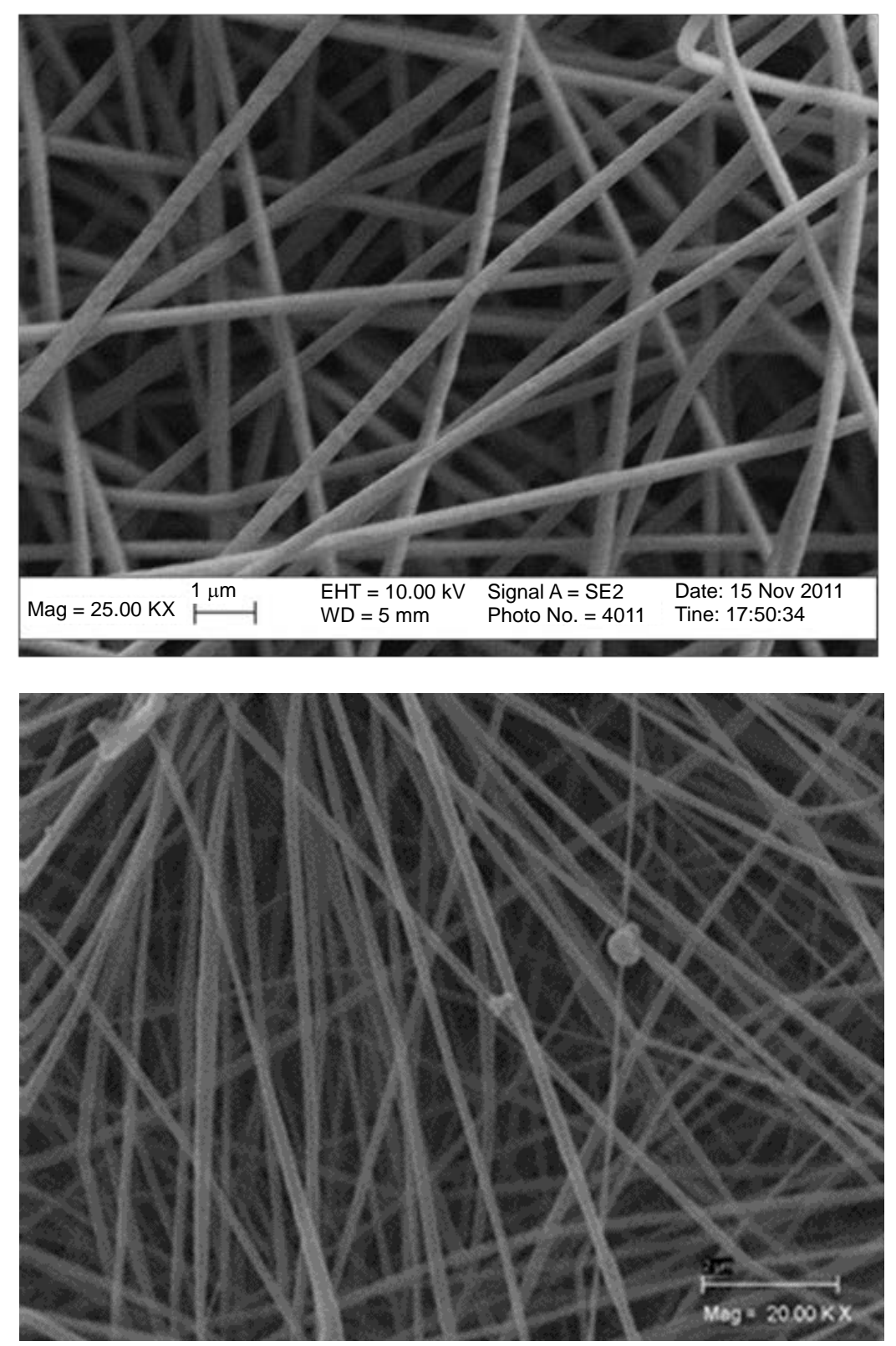

Figure 4. SEM images of $\mathrm{P}$ (AN-co-VAc) (upper-scale bar is 1 micron) $\mathrm{P}$ (AN-coVAc)/PPy2, 0.045 mole\% (lower-scale bar is 2 micron). 


\section{Conclusions}

The viscosity of the solution has a significant effect on $\mathrm{P}$ (AN-co-VAc) electrospinning conditions and the nanofiber diameter. The average diameters of $\mathrm{P}$ (AN-co-VAc) nanofibers decrease from 445 to $130 \mathrm{~nm}$ with increasing feed ratio of VAc weight percent. The $\mathrm{P}$ (AN-co-VAc) with $10 \mathrm{wt} \%$ of VAc feed ratio is resulted from the highest average nanofiber diameter. The $\mathrm{P}$ (AN-co-VAc) electrospun nanofiber mats, especially the copolymer which has the feed ratio of $30 \mathrm{wt} \%$ VAc can be used as a nanofiber membrane in filtration and as a carbon nanofiber precursor for energy storage applications due to high surface to volume ratio, high thermal stability, homogeneous, and thinner nanofiber distribution. The diameters of the fibers generally decrease slightly as the content of PPy is increased and bead-free and smaller nanofibers are obtained for the polymer solutions having low polymer content. The high degree of homogeneity and molecular order induced by molecular dispersion of conjugated polymer on copolymer matrix without phase separation can improve the transport properties and stability of conjugating polymers, which are critical for high-performance organic electronics.

These fibers can be used as textile filtration nanofiber mats and for carbon nanofiber precursor in energy storage applications.

\section{References}

[1] Ghalib, H., Abdullah, I. and Daik, R. (2013) Electrically Conductive Polystyrene/Polypyrrole Nanocomposites Prepared via Emulsion Polymerization. Polymer-Plastics Technology and Engineering, 52, 478-484. http://dx.doi.org/10.1080/03602559.2012.758736

[2] Cetiner, S., Sen, S. and Arman, B. (2013) Acrylonitrile/Vinyl Acetate Copolymer Nanofibers with Different Vinylacetate Content. Journal of Applied Polymer Science, 127, 3830-3838. http://dx.doi.org/10.1002/app.37690

[3] Cetiner, S., Kalaoglu, F., Karakas, H. and Sarac, A.S. (2011) Preparation and Characterization of Conductive Poly (Acrylonitrile-Co-Vinyl Acetate) Composite Films: Matrix Polymerization of Pyrrole Derivatives. Fibers and Polymers, 12, 151-158.

[4] Cetiner, S., Kalaoglu, F., Karakas, H. and Sarac, A.S. (2011) Dielectric, FTIR Spectroscopic and Atomic Force Microscopic (AFM) Studies on Polypyrrole-Poly (Acrylonitrile-Co-Vinyl Acetate) Composites. Polymer Composites, 32, 546-557. http://dx.doi.org/10.1002/pc.21075

[5] Sahoo, N.G., Jung, Y.C., So, H.H. and Cho, J.W. (2007) Polypyrrole Coated Carbon Nanotubes: Synthesis, Characterization, and Enhanced Electrical Properties. Synthetic Metals, 157, 374-379. http://dx.doi.org/10.1016/j.synthmet.2007.04.006

[6] Chen, C.-L., Teng, H. and Lee, Y.-L. (2011) Preparation of Highly Efficient Gel-State Dye-Sensitized Solar Cells Using Polymer Gel Electrolytes Based on Poly (Acrylonitrile-Co-Vinyl Acetate). Journal of Materials Chemistry, 21, 628-632. http://dx.doi.org/10.1039/c0jm03597a

[7] Chen, C.-L., Teng, H. and Lee, Y.-L. (2011) In Situ Gelation of Electrolytes for Highly Efficient Gel-State Dye-Sensitized Solar Cells. Advanced Materials, 23, 4199-4204. http://dx.doi.org/10.1002/adma.201101448

[8] Xia, Y. and Lu, Y. (2011) One-Step Fabrication and Further Modification of Poly (Acrylonitrile-Co-Vinyl Acetate) Microsphere. Journal of Polymer Research, 18, 1645-1651. http://dx.doi.org/10.1007/s10965-011-9569-7

[9] Xiao, H. and Xia, Y. (2010) Poly (Acrylonitrile-Co-Vinyl Acetate)/Ag Composite Microspheres: One-Pot Fabrication and Application as Catalyst. Polymer Engineering \& Science, 50, 1767-1772. http://dx.doi.org/10.1002/pen.21708

[10] Cetiner, S., Olariu, M., Ciobanu, R.C., Karakas, H., Kalaoglu, F. and Sarac, A.S. (2010) Polypyrrole-Polyacrylonitrile Composite Films: Dielectric, Spectrophotometric and Morphologic Characterization. Fibers and Polymers, 11, 843850. http://dx.doi.org/10.1007/s12221-010-0843-9

[11] Cetiner, S., Karakas, H., Ciobanu, R.C., Olariu, M., Kaya, N.U., Unsal, C., Kalaoglu, F. and Sarac, A.S. (2010) Polymerization of Pyrrole Derivatives on Polyacrylonitrile Matrix, FTIR-ATR and Dielectric Spectroscopic Characterization of Composite Thin Films. Synthetic Metals, 160, 1189-1196. http://dx.doi.org/10.1016/j.synthmet.2010.03.007

[12] Vishhuvardhan, T.K., Kulkarni, V.R., Basavaraja, C. and Raghavendra, S.C. (2006) Synthesis, Characterization and a.c. Conductivity of Polypyrrole/ $\mathrm{Y}_{2} \mathrm{O}_{3}$ Composites. Bulletin of Materials Science, 29, 77-83.

http://dx.doi.org/10.1007/BF02709360 